\title{
Pretreatment with Ranitidine Bismuth Citrate May Improve Success Rates of Helicobacter pylori Eradication: A Prospective, Randomized, Controlled and Open-Label Study
}

\author{
Jin Young Yoon, ${ }^{1}$ Min Seob Kwak, ${ }^{1}$ Jung Won Jeon ${ }^{1}$ and Jae Myung Cha ${ }^{1}$ \\ ${ }^{1}$ Department of Internal Medicine, Division of Gastroenterology, Kyung Hee University Hospital at Gang Dong, \\ Seoul, Korea
}

\begin{abstract}
Effective Helicobacter pylori $(H$. pylori) eradication is a major public health concern; however, eradication failure rates with the standard triple therapy remain high. We aimed to investigate the effectiveness and tolerability of ranitidine bismuth citrate $(\mathrm{RBC})$ pretreatment before standard triple therapy for $H$. pylori eradication. A prospective, randomized, controlled, and open-label clinical trial was conducted from June to December 2019. H. pylori eradication rate, safety, and tolerability were compared between the standard treatment group (esomeprazole, amoxicillin, and clarithromycin for 7 days) and RBC pretreatment group (RBC for 2 weeks before standard triple therapy). This trial ended earlier than estimated owing to the $\mathrm{N}$-nitrosodimethylamine concerns with ranitidine. Success rates of $H$. pylori eradication were $80.9 \%$ and $67.3 \%$ in the RBC pretreatment $(n=47)$ and standard treatment $(n=52)(p=0.126)$ groups, respectively. Our trial was discontinued earlier than planned; however, a statistical significance would be achieved by expansion of our data $(p=0.031)$ if patient enrollment numbers reached those initially planned. Adverse event rates were comparable between groups $(25.5 \%$ in the pretreatment group vs. $28.8 \%$ in the standard treatment group), without serious event. Tolerability was excellent in both groups, recorded as $97.9 \%$ and $100 \%$ in the pretreatment and standard treatment groups, respectively. Compared with the standard triple regimen, RBC pretreatment for 2 weeks may achieve higher $H$. pylori eradication rates, with excellent safety and tolerability. However, this study necessitates further validation as it was discontinued early owing to the $\mathrm{N}$-nitrosodimethylamine issues of ranitidine.
\end{abstract}

Keywords: eradication rate; Helicobacter pylori; N-nitrosodimethylamine; ranitidine bismuth citrate; pretreatment Tohoku J. Exp. Med., 2021 September, 255 (1), 41-48.

\section{Introduction}

Helicobacter pylori (H. pylori) is a major human infection occurring through colonization of the stomach in approximately half the global population (Hooi et al. 2017). H. pylori has been known as a causative factor for the development of several upper gastrointestinal diseases, including functional dyspepsia, gastritis, peptic ulcer, gastric adenocarcinoma, and mucosa-associated lymphoid tissue lymphoma (Malfertheiner et al. 2017). Moreover, its association with other organ diseases has been investigated, including colonic and pancreatic malignancies, hematologic diseases such as immune thrombocytopenic purpura, vitamin B12 deficiency and refractory iron deficiency anemia, vascular diseases such as coronary heart, and cerebrovascu- lar disease including Parkinson's disease (Trikudanathan et al. 2011; Banic et al. 2012; Chen et al. 2013; Sonnenberg and Genta 2013). As the eradication of H. pylori can ameliorate clinical consequences, efficacious $H$. pylori eradication continues to be a major public health concern (Jonaitis et al. 2018).

To date, the most widely recommended regimen as first-line eradication therapy for $H$. pylori is the standard triple therapy, consisting of a proton-pump inhibitor (PPI), clarithromycin, and amoxicillin (Kim et al. 2014; Malfertheiner et al. 2017). However, concerns regarding eradication failure with standard triple therapy have been expressed (Malfertheiner et al. 2017). Previously, studies have reported that eradication rates with standard triple therapy have possibly decreased from $93.5 \%$ in 2003 to

Received April 20, 2021; revised and accepted June 23, 2021. Published online September 16, 2021; doi: 10.1620/tjem.255.41.

Correspondence: Jae Myung Cha, M.D., Ph.D., Department of Internal Medicine, Kyung Hee University Hospital at Gangdong, Kyung

Hee University School of Medicine, 892 Dongnam-ro, Gangdong-gu, Seoul 05278, Republic of Korea.

e-mail:drcha@khu.ac.kr

(C)2021 Tohoku University Medical Press. This is an open-access article distributed under the terms of the Creative Commons Attribution-NonCommercial-NoDerivatives 4.0 International License (CC-BY-NC-ND 4.0). Anyone may download, reuse, copy, reprint, or distribute the article without modifications or adaptations for non-profit purposes if they cite the original authors and source properly.

https://creativecommons.org/licenses/by-nc-nd/4.0/ 
69.3\% in 2015 (Kim et al. 2015; Jung et al. 2017). Notably, eradication success rates could be decreased by elevated intragastric bacterial load before treatment, insufficient acid control in the stomach, nonadherence to drug regimens, and host genetic polymorphisms involved in the metabolism of PPI, as well as antibiotic resistance as the most crucial governing factor (Labenz et al. 1995; Jenks 2002).

To improve success rates of $H$. pylori eradication, most previous studies have attempted to avoid antibiotic resistance by altering antibiotic composition or duration (Malfertheiner et al. 2017, O'Connor et al. 2019). However, lowering the intragastric bacterial load before treatment or sufficient control of gastric acidity has received minimal attention. Pretreatment with ranitidine bismuth citrate (RBC) before initiating $H$. pylori eradication therapy may achieve these purposes as this compound has dual effects, the gastric anti-secretory activity of ranitidine and the mucosal protective, anti-pepsin, and anti-H. pylori properties of bismuth (Stables et al. 1993; Gisbert et al. 2005). However, no previous study has investigated the synergic effect of RBC pretreatment on the H. pylori eradication therapy.

In this prospective, randomized, controlled, and openlabel study, we aimed to investigate the efficacy and tolerability of a 2-week RBC pretreatment before standard triple therapy compared with standard triple therapy without pretreatment in patients with $H$. pylori infection.

\section{Materials and Methods}

\section{Study population}

Patients were eligible if aged between 20 and 70 years and confirmed presenting $H$. pylori infection within one year using the ${ }^{13} \mathrm{C}$-urea breath test (UBT), campylobacterlike organism test, or histological examination (Giemsa or hematoxylin and eosin stain). Patients were excluded if they: 1) had used nonsteroidal anti-inflammatory drugs, corticosteroids, antimicrobials, acid-suppressing medications (such as PPIs and $\mathrm{H}_{2}$-blockers), or bismuth compounds, less than two weeks before the screening visit; 2) had a previous gastric malignancy; 3) demonstrated alarming signs (e.g., abnormal weight loss, hematochezia, anemia, or significant bowel habit changes); 4) presented uncontrolled comorbidities; 5) were pregnant or breastfeeding; 6) were drug users or alcoholics. All enrolled patients received comprehensive information regarding the study, with informed consent obtained before the initiation of any study-related processes. The study protocol was reviewed and approved by the Institutional Review Board (IRB) of our hospital (KHNMC IRB 2018-05-022) and investigations were performed in accordance with the Declaration of Helsinki and Good Clinical Practice guidelines. The study was registered at the WHO International Clinical Trial Registry Platform (list on https://apps.who.int/trialsearch/ or https://cris.nih.go.kr) using the identifier KCT0005871.

\section{Study design}

This prospective, randomized, controlled, and open- label clinical trial was conducted at Kyung Hee University Hospital at Gangdong, from June to December 2019. After screening for inclusion and exclusion criteria, enrolled patients were assigned consecutive allocation numbers, matched at a 1:1 ratio to a randomization code through a table of random numbers. The standard treatment group was defined as $H$. pylori eradication using the first-line triple 7-day regimen (esomeprazole $40 \mathrm{mg}$ b.i.d. + amoxicillin 1,000 mg b.i.d. + clarithromycin $500 \mathrm{mg}$ b.i.d.) (Kim et al. 2014). The pretreatment group was defined as pretreatment medication with RBC (Albis D ${ }^{\circledR}$, Daewoong Pharmaceutical Co., Ltd., Seoul, Korea), which included ranitidine hydrochloride $168 \mathrm{mg}$ (150 mg as ranitidine), tripotassium bismuth dicitrate $200 \mathrm{mg}$, and sucralfate hydrate $600 \mathrm{mg}$, initiated before the same first-line triple 7-day regimen. Patients were randomized into either the standard treatment group (standard triple regimen only) or pretreatment group (RBC administration twice daily for 2 weeks before the start of standard triple regimen). Successful $H$. pylori eradication was evaluated using ${ }^{13} \mathrm{C}$-UBT at 6-8 weeks after the completion of the standard triple regimen. Briefly, $50 \mathrm{mg}$ ${ }^{13} \mathrm{C}$-urea was dissolved in water and administered orally. Baseline and 30-min breath samples were assayed using an infrared spectrometer producing computer-generated results. Negative results were defined as a computer-generated $\delta^{13} \mathrm{CO}_{2}$ value $<2 \%$, with a positive result $\geq 2 \%$ considered as treatment failure (Ferwana et al. 2015). During the study period, patients recorded the time at which the study product was administered, as well as the occurrence of any adverse events, in daily diaries. All unused products were returned to the study site, and compliance was calculated at the last visit. Compliance was evaluated by counting the pills returned by patients and was deemed poor compliance if the intake was $<90 \%$ of the drugs prescribed (Kotilea et al. 2017).

\section{Statistical analysis}

The primary outcome was the success rates of $H$. pylori eradication after completion of the entire regimen. Based on a previous study in a similar setting, the sample size was calculated assuming an $85 \%$ eradication rate as the primary endpoint in the pretreatment group and $70 \%$ in the control group (Tokoro et al. 2011; Kim et al. 2015). We estimated that a sample size of 120 patients per group would have a statistical power of $80 \%$ and a two-sided $\alpha$-risk of 0.05 . Initially, we planned to enroll 126 subjects in each group, assuming a 5\% dropout rate. Efficacy was assessed by per-protocol (PP), and safety by intention to treat analysis (ITT). ITT analysis included all participants who had administered any medication.

Continuous variables are presented as means \pm standard deviations (SD) for clinical data and were compared using two-sample t-tests. Categorical variables are presented as numbers and percentages and were compared using the Chi-squared test or Fisher's exact test. All statistical tests were two-sided, and a p-value $<0.05$ was consid- 
ered statistically significant. All statistical analyses were performed using SPSS for Windows release 18.0 (SPSS, Inc., Chicago, IL, USA).

\section{Results}

This trial ended earlier than initially planned owing to the N-nitrosodimethylamine (NDMA) issue raised by the Food and Drug Administration (FDA) of the United States as our pretreatment $\mathrm{RBC}$ included ranitidine. During our clinical trial, the FDA (2019) reported that ranitidine contains a low level of nitrosamine impurity called NDMA, which is a known environmental contaminant and classified as a probable human carcinogen (Zantac). As the FDA requested the removal of all ranitidine products from the market and the Korean FDA limited the sale of 269 therapeutic agents containing ranitidine in January 2020, we decided to discontinue the present trial after reporting to the Institutional Review Board as a significant adverse drug reaction. Thus, we could not achieve the sample size goal: we enrolled only $114(45.2 \%)$ subjects despite initially intending to enroll 252 subjects (126 subjects in each group).

\section{Baseline characteristics}

In total, 114 subjects were invited to participate in this study, and 9 subjects were ineligible as they failed to meet the inclusion criteria. Overall, 105 subjects consented and were enrolled in the study, followed by random allocation to the pretreatment group $(\mathrm{n}=52)$ or control group $(\mathrm{n}=$ 53). After allocation, five subjects were excluded from the pretreatment group owing to consent withdrawal $(n=4)$ and poor compliance $(\mathrm{n}=1)$. Additionally, one subject in the control group was excluded owing to a newly diagnosed malignancy. Finally, 99 subjects (47 subjects in the pretreatment group and 52 subjects in the control group) were analyzed (Fig. 1). The baseline characteristics of participants did not differ between the two groups regarding age, sex, alcohol consumption, smoking, comorbidities, exercise frequency, findings on gastroduodenal endoscopy, and medication use (Table 1).

\section{Clinical outcomes}

Overall, the H. pylori eradication rate was $73.7 \%$ (73/99). Success rates of $H$. pylori eradication were $80.9 \%$ and $67.3 \%$ in the RBC pretreatment and control groups, respectively, as only $9(19.1 \%)$ of the 47 subjects in the $\mathrm{RBC}$ pretreatment group and $17(32.7 \%)$ in the triple standard regimen group demonstrated eradication failure (Fig. 2). As our trial was discontinued earlier than planned, the H. pylori eradication rate failed to attain statistical significance between the pretreatment and control groups $(\mathrm{p}=$ 0.126). No significant differences were observed between the success and failure groups of $H$. pylori eradication in terms of age, sex, alcohol consumption, smoking, exercise pattern, presence of comorbidity, or aspirin use (Table 2).

\section{Safety and tolerability}

In ITT analysis, 105 subjects were included to evaluate the safety profile (Table 3). Adverse event rates were comparable between the two groups without a significant difference $(25.5 \%$ in the pretreatment group vs. $28.8 \%$ in the standard treatment group). All drug-related adverse events were mild in intensity with no serious event reported; however, one subject in the standard treatment group was unex-

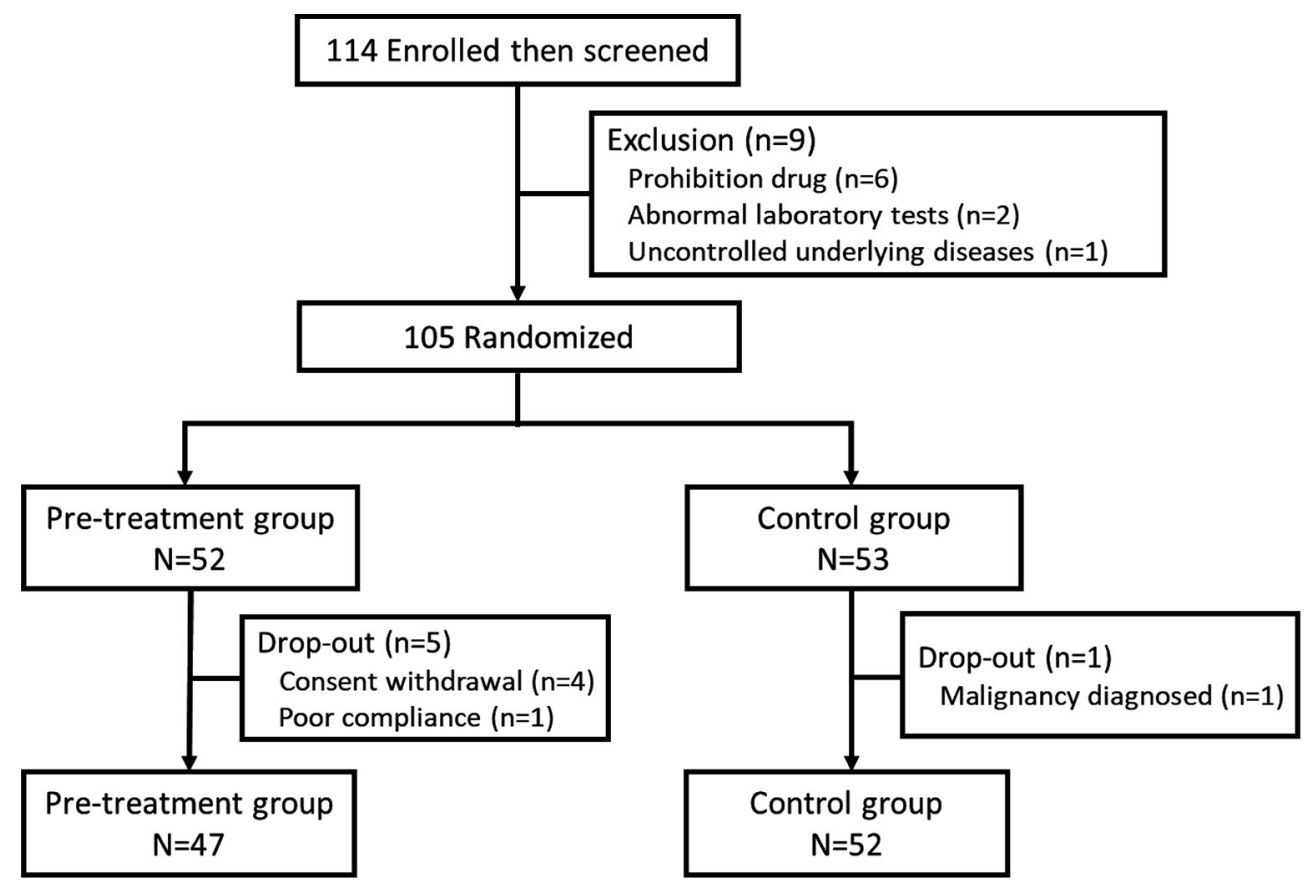

Fig. 1. Patient flow diagram. 
Table 1. Baseline characteristics of study subjects.

\begin{tabular}{|c|c|c|c|}
\hline & $\begin{array}{l}\text { Pre-treatment } \\
\text { group } \\
(\mathrm{n}=47)\end{array}$ & $\begin{array}{l}\text { Standard treatment } \\
\text { group } \\
(\mathrm{n}=52)\end{array}$ & p-value \\
\hline Age & $56.2 \pm 9.9$ & $53.5 \pm 12.4$ & 0.240 \\
\hline Sex, n (\%) & & & 0.287 \\
\hline Male & $24(51.1)$ & $21(40.4)$ & \\
\hline Female & $23(48.9)$ & $31(59.6)$ & \\
\hline Alcohol consumption, $\mathrm{n}(\%)$ & & & 0.114 \\
\hline No & $26(55.3)$ & $38(73.1)$ & \\
\hline Social & $17(36.2)$ & $13(25.0)$ & \\
\hline Heavy & $4(8.5)$ & $1(1.9)$ & \\
\hline \multicolumn{4}{|l|}{ Smoking, n (\%) } \\
\hline No & $40(85.1)$ & $45(86.5)$ & 0.838 \\
\hline Yes & $7(14.9)$ & $7(13.5)$ & \\
\hline Exercise, n (\%) & & & 0.295 \\
\hline No & $15(31.9)$ & $19(36.5)$ & \\
\hline $1-3$ times/week & $24(51.1)$ & $19(36.5)$ & \\
\hline$\geq 4$ times/week & $8(17.0)$ & $14(26.9)$ & \\
\hline Findings on gastroduodenal endoscopy & & & 0.595 \\
\hline Erosion & $16(34.0)$ & $11(21.2)$ & \\
\hline Gastric or Duodenal ulcer & $7(14.9)$ & $7(13.5)$ & \\
\hline Benign gastric polyp & $4(8.5)$ & $1(1.9)$ & \\
\hline Intestinal metaplasia & $24(51.1)$ & $23(44.2)$ & \\
\hline \multicolumn{4}{|l|}{ Comorbidity, n (\%) } \\
\hline Hypertension & $10(21.3)$ & $9(17.3)$ & 0.617 \\
\hline Diabetes mellitus & $1(2.1)$ & $4(7.7)$ & 0.207 \\
\hline Thyroid disease & $0(0.0)$ & $3(5.8)$ & 0.094 \\
\hline Dyslipidemia & $3(6.4)$ & $3(5.8)$ & 0.898 \\
\hline Aspirin users, $\mathrm{n}(\%)$ & $1(2.1)$ & $3(5.8)$ & 0.358 \\
\hline
\end{tabular}

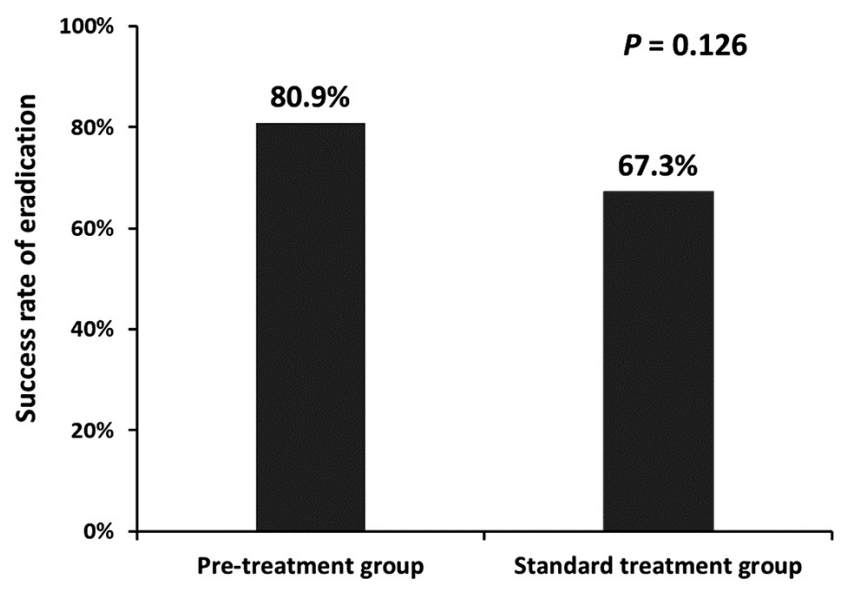

Fig. 2. Success rate of Helicobacter pylori eradication. Pre-treatment group was administered with ranitidine bismuth citrate for 2 weeks before initiating standard treatment of triple 7-day regimen for $H$. pylori eradication. Standard treatment group was administered only with triple 7-day regimen (esomeprazole $40 \mathrm{mg}$ b.i.d. + amoxicillin $1,000 \mathrm{mg}$ b.i.d. + clarithromycin $500 \mathrm{mg}$ b.i.d.). pectedly diagnosed with lung cancer during the study period, resulting in trial termination in this patient. In both groups, tolerability was excellent, recorded as $97.9 \%$ in the pretreatment group, and $100 \%$ in the standard treatment group. In the pretreatment group, one subject quit administering the standard regimen for $H$. pylori eradication on the second day owing to halitosis, nausea, and vomiting after completion of the pretreatment regimen.

\section{Discussion}

Unfortunately, we encountered NDMA issues and decided to discontinue this study with the interim data only. Our study demonstrated the $H$. pylori eradication rate was $67.3 \%$ in the standard treatment group (7-days standard triple therapy containing PPI, clarithromycin, and amoxicillin) and $80.9 \%$ in the pretreatment group (RBC pretreatment for 2-weeks before standard triple therapy) in the PP analysis. Although the RBC pretreatment group seemed more effective, the difference between the two groups did not attain statistical significance owing to the insufficient sample size $(p=0.126)$. If the number of enrolled patients reached initial planning presenting the same pattern, a sta- 
Table 2. Clinical characteristics of study subjects according to the success or failure of eradication therapy.

\begin{tabular}{|c|c|c|c|}
\hline & $\begin{array}{l}\text { Success group } \\
\quad(\mathrm{n}=73)\end{array}$ & $\begin{array}{l}\text { Failure group } \\
\quad(\mathrm{n}=26)\end{array}$ & p-value \\
\hline Age & $55.0 \pm 11.8$ & $54.2 \pm 10.1$ & 0.768 \\
\hline Sex, n $(\%)$ & & & 0.196 \\
\hline Male & $36(49.3)$ & $9(34.6)$ & \\
\hline Female & $37(50.7)$ & $17(65.4)$ & \\
\hline Alcohol consumption, $\mathrm{n}(\%)$ & & & 0.142 \\
\hline No & $44(60.3)$ & $20(76.9)$ & \\
\hline Social & $26(35.6)$ & $4(15.4)$ & \\
\hline Heavy & $3(4.1)$ & $2(7.7)$ & \\
\hline \multicolumn{4}{|l|}{ Smoking, n (\%) } \\
\hline No & $60(82.2 \%)$ & $25(96.2 \%)$ & 0.105 \\
\hline Yes & $13(17.8 \%)$ & $1(3.8 \%)$ & \\
\hline Exercise, n (\%) & & & 0.057 \\
\hline No & $30(41.1)$ & $4(15.4)$ & \\
\hline 1-3 times/week & $29(39.7)$ & $14(53.8)$ & \\
\hline$\geq 4$ times/week & $14(19.2)$ & $8(30.8)$ & \\
\hline Comorbidity, n (\%) & & & 0.343 \\
\hline No & $52(71.2)$ & $21(80.8)$ & \\
\hline Yes & $21(28.8)$ & $5(19.2)$ & \\
\hline Treatment group, n (\%) & & & 0.126 \\
\hline Pretreatment with RBC & $38(52.1)$ & $9(34.6)$ & \\
\hline Standard treatment & $35(47.9)$ & $17(65.4)$ & \\
\hline Aspirin users, $\mathrm{n}(\%)$ & & & 1.000 \\
\hline No & $70(95.9)$ & $25(96.2)$ & \\
\hline Yes & $3(4.1)$ & $1(3.8)$ & \\
\hline
\end{tabular}

RBC, Ranitidine bismuth citrate.

Table 3. Adverse events reported over the entire treatment period through intention-to-treat analysis.

\begin{tabular}{lccc}
\hline & $\begin{array}{c}\text { Pre-treatment group } \\
(\mathrm{n}=52)\end{array}$ & $\begin{array}{c}\text { Standard treatment } \\
\text { group }(\mathrm{n}=53)\end{array}$ & p-value \\
\hline Gastrointestinal disorder, n (\%) & $3(5.8)$ & $2(3.8)$ & 0.653 \\
$\quad$ Nausea/Vomiting & $2(3.8)$ & $4(7.5)$ & \\
$\quad$ Diarrhea & $4(7.7)$ & $5(9.4)$ & 1.000 \\
$\quad$ Dyspepsia & & & \\
Infection/Inflammation & $1(1.9)$ & $0(0.0)$ & 1.000 \\
$\quad$ Myalgia & $0(0.0)$ & $2(3.8)$ & \\
$\quad$ URI-related symptoms & & $1(1.9)$ & \\
Others & $0(0.0)$ & $1(1.9)$ & \\
Lung cancer & $2(3.8)$ & & \\
Halitosis &
\end{tabular}

URI, Upper respiratory infection.

tistical significance would be achieved by duplication of the same data $(p=0.031)$. In the present study, the most important finding is that $\mathrm{RBC}$ pretreatment could improve the eradication rate achieved with the standard triple therapy.
PPI pretreatment before $H$. pylori eradication can be considered to decrease intragastric acidity and to influence H. pylori eradication rate. However, previous observations with PPI pretreatment before $H$. pylori eradication have been disappointing (Labenz et al. 1993; Bayerdorffer et al. 
1995; Janssen et al. 2005; Yoon et al. 2014). A meta-analysis has reported that no significant difference can be observed in the $H$. pylori eradication rates using triple and quadruple therapies regardless of PPI pretreatment (Janssen et al. 2005). Additionally, long-term PPI pretreatment for over 8 weeks fails to significantly alter the $H$. pylori eradication rates (Yoon et al. 2014). Furthermore, another two studies have reported that PPI pretreatment significantly lowers H. pylori eradication rates (Labenz et al. 1993; Bayerdorffer et al. 1995). Despite conflicting data regarding PPI pretreatment for $\mathrm{H}$. pylori eradication, this policy is currently discouraged as it remains certain that PPI pretreatment has no beneficial effects (Ierardi et al. 2019). The negative effects of PPI pretreatment on eradication rates might be explained by the transformation of $H$. pylori from spiral to coccoid form (Andersen et al. 2000). Reportedly, an in vitro study has observed that PPI activity is directly associated with the viability and number of coccoid forms, and absence of PPI in the culture medium induces an increases transformation into spiral forms, as well as viability (Saniee et al. 2016). The coccoid form of H. pylori is associated with the capacity to avoid immune system detection and promotes therapeutic failure (Krzyzek and Grande 2020).

Conversely, the $\mathrm{H}_{2}$-receptor antagonist (H2RA) has anti-secretory ability but no intrinsic antimicrobial effect (Graham et al. 2003; Tokoro et al. 2011). As H2RA does not influence $H$. pylori transformation, theoretically, H2RA pretreatment might be more suitable to improve the $H$. pylori eradication efficacy than PPI. A retrospective study has observed that the H2RA pretreatment group achieved a marginally higher $H$. pylori eradication rate when compared to the standard triple therapy group $(81.6 \%$ vs. $77.6 \%, \mathrm{p}=$ 0.3799) (Tokoro et al. 2011), suggesting the possibility of H2RA pretreatment for $H$. pylori eradication; however, it was limited by the retrospective nature, including heterogeneous $H$. pylori eradication regimen, various durations (1-150 days) of H2RA pretreatment, and diverse kinds and dosages of H2RA. Thus, the previous retrospective study is insufficient to evaluate the effect of H2RA pretreatment on H. pylori eradication efficacy. Our prospective trial selected $\mathrm{RBC}$, composed of ranitidine, bismuth, and sucralfate, as the pretreatment medication as it could optimally maximize the effectiveness of pretreatment before $H$. pylori eradication. Ranitidine reduces intragastric acidity and bismuth possesses direct bactericidal effects on H. pylori mediated by inhibition of bacterial enzymes, adenosine triphosphate synthesis, and adherence of the bacteria to the gastric mucosa (Alkim et al. 2017). Furthermore, RBC demonstrates significantly greater solubility especially, at lower $\mathrm{pH}$ values; this property is highly relevant to the enhanced dual function that low dose RBC could produce therapeutic effects when compared to the insoluble simple admixture of bismuth citrate with ranitidine (McColm et al. 1996).

In our study, the eradication rate in the standard treatment group for 7 days was only $67.3 \%$, which was consis- tent with a recent decreasing trend in eradication rates. Recently, three studies have identified H. pylori eradication rates for the triple standard regimen in Korea. Reportedly, H. pylori eradication rates have significantly decreased from $84.9 \%-87.5 \%$ in $2001-2007$ to $80.0 \%-81.4 \%$ in 2008 2010 (Shin et al. 2016). Over the last 10 years, annual eradication rates have gradually decreased from $93.5 \%$ in 2003 to $78.8 \%$ in 2012 (Kim et al. 2015). In a recent network meta-analysis, $H$. pylori eradication rates of triple standard regimen for 7 days were $72.6 \%, 64.3 \%, 69.3 \%$, and $70.8 \%$ in $2013,2014,2015$, and 2016 , respectively (Jung et al. 2017). The previous three studies were limited owing to a retrospective or meta-analysis design; therefore, our finding presents the most reliable result of the $H$. pylori eradication rate from prospective data. Furthermore, we concurred with evidence that $H$. pylori eradication rates continue to decrease in 2019.

Globally, the efficacy of $H$. pylori eradication with clarithromycin-based regimens has decreased in parallel with increasing clarithromycin resistance (Savoldi et al. 2018), as clarithromycin resistance is the strongest factor affecting the $H$. pylori eradication rate with the triple standard regimen (Malfertheiner et al. 2017). In Korea, recent clarithromycin resistance rate has been known as $23.4 \%$ (Lee et al. 2013). Accordingly, alarming rates of clarithromycin resistance have resulted in insufficient eradication rates following conventional triple regimen in South Korea (Gong et al. 2014; Shin et al. 2016). Hence, standard triple therapy possesses no additional qualifications as the firstline regimen, and clarithromycin-containing triple regimen without susceptibility test should be abandoned in regions where the clarithromycin resistance rate exceeds $15 \%$ (Malfertheiner et al. 2017). A success rate of at least $80 \%$ is an essential requirement for an acceptable first-line regimen for $H$. pylori eradication (Graham and Fischbach 2010). Hence, current guidelines have recommended modifying antibiotic regimens such as bismuth-based quadruple, concomitant or sequential therapy, and antibiotic susceptibility tests (Kim et al. 2014; Malfertheiner et al. 2017; O'Connor et al. 2019). However, it remains challenging to apply a uniform $H$. pylori eradication because antibiotic resistance differs geographically and individually. Our study demonstrated that RBC pretreatment for 2-weeks increased the eradication rate observed with triple standard therapy. RBC pretreatment could improve the $H$. pylori eradication rate as substantially as changing the antibiotic regimen because it could improve the stability of antibiotics in the stomach, rendering them sensitive to $H$. pylori. Notably, our proposal could be applied to entire regions regardless of antibiotic resistance.

Patient adherence to treatment is an important factor to predict the successful eradication of $H$. pylori (Bang and Baik 2014). In this study, most patients demonstrated good adherence to the whole treatment regimen and only one patient discontinued the triple standard therapy in the pretreatment group. Although there exists a risk for develop- 
ing tolerance during $\mathrm{RBC}$ pretreatment for 2 weeks before H. pylori eradication, we assumed that the tolerance to the regimen would not pose a major problem as no patient violated the study regimen in this study. Furthermore, no significant predictor was observed for eradication failure among host characteristics, differing from the previous study reporting female gender, alcohol consumption and smoking as predictors of eradication failure (Kim et al. 2015; Chey et al. 2017). The reason why smoking and alcohol habits had no negative influence on $H$. pylori eradication might be caused by the limited number of the patients with current smokers (14\%) or heavy alcoholics (5\%) from this point of view.

The major limitation of this study is that we could not complete recruiting the planned number of participants owing to NDMA issues associated with ranitidine. Although patients participated in our study at that time had safety concern about carcinogenic NDMA, recent reports rediscovered that ranitidine user with exposure to NDMA did not increase the risk of overall cancer in cohort studies (Kantor et al. 2021; Yoon et al. 2021). Furthermore, we failed to evaluate antibiotic resistance of $H$. pylori, including clarithromycin resistance. Because the beneficial effect of bismuth on first-line eradication become noticeable in a high prevalence of clarithromycin resistance (Ko et al. 2019), we had limitation to identify which component of $\mathrm{RBC}$ played more major role on our superior cure rate in pretreatment group without antibiotics susceptibility. However, in clinical settings, the susceptibility test has been used with limited transferability across countries and populations, presenting cost implications. Hence, it would be ideal to include whole populations without antibiotic resistance H. pylori to investigate eradication.

In conclusion, RBC pretreatment for 2 weeks may achieve higher $H$. pylori eradication rates when compared with the standard triple regimen, with excellent safety and tolerability. However, our findings should be validated as this study was discontinued early with interim data owing to NDMA issues.

\section{Conflict of Interest}

The authors declare no conflict of interest.

\section{Author Contributions}

Study concept and design: Jin Young Yoon and Jae Myung Cha. Patient enrollment and data acquisition: Jin Young Yoon, Jung Won Jeon and Jae Myung Cha. Data analysis and interpretation: Jin Young Yoon and Min Seob Kwak. Obtained funding: Jae Myung Cha. Drafting of the manuscript: Jin Young Yoon.

\section{References}

Alkim, H., Koksal, A.R., Boga, S., Sen, I. \& Alkim, C. (2017) Role of bismuth in the eradication of Helicobacter pylori. Am. J. Ther., 24, e751-e757.

Andersen, L.P., Dorland, A., Karacan, H., Colding, H., Nilsson,
H.O., Wadstrom, T. \& Blom, J. (2000) Possible clinical importance of the transformation of Helicobacter pylori into coccoid forms. Scand. J. Gastroenterol., 35, 897-903.

Bang, C.S. \& Baik, G.H. (2014) Attempts to enhance the eradication rate of Helicobacter pylori infection. World J. Gastroenterol., 20, 5252-5262.

Banic, M., Franceschi, F., Babic, Z. \& Gasbarrini, A. (2012) Extragastric manifestations of Helicobacter pylori infection. Helicobacter, 17 Suppl 1, 49-55.

Bayerdorffer, E., Miehlke, S., Mannes, G.A., Sommer, A., Hochter, W., Weingart, J., Heldwein, W., Klann, H., Simon, T., Schmitt, W., Bästlein, E., Eimiller, A., Hatz, R., Lehn, N., Dirschedl, P., Stolte, M. et al. (1995) Double-blind trial of omeprazole and amoxicillin to cure Helicobacter pylori infection in patients with duodenal ulcers. Gastroenterology, 108, 1412-1417.

Chen, B.F., Xu, X., Deng, Y., Ma, S.C., Tang, L.Q., Zhang, S.B. \& Chen, Z.F. (2013) Relationship between Helicobacter pylori infection and serum interleukin-18 in patients with carotid atherosclerosis. Helicobacter, 18, 124-128.

Chey, W.D., Leontiadis, G.I., Howden, C.W. \& Moss, S.F. (2017) ACG clinical guideline: treatment of Helicobacter pylori infection. Am. J. Gastroenterol., 112, 212-239.

FDA (US Food and Drug Administration) (2019) Zantac (ranitidine): Safety Information - NDMA Found in Samples of Some Ranitidine Medicines; c2019.

https://www.fda.gov/safety/medical-product-safety-information/ zantac-ranitidine-safety-information-ndma-found-samplessome-ranitidine-medicines [Accessed: April 18, 2020].

Ferwana, M., Abdulmajeed, I., Alhajiahmed, A., Madani, W., Firwana, B., Hasan, R., Altayar, O., Limburg, P.J., Murad, M.H. \& Knawy, B. (2015) Accuracy of urea breath test in Helicobacter pylori infection: meta-analysis. World J. Gastroenterol., 21, 1305-1314.

Gisbert, J.P., Gonzalez, L. \& Calvet, X. (2005) Systematic review and meta-analysis: proton pump inhibitor vs. ranitidine bismuth citrate plus two antibiotics in Helicobacter pylori eradication. Helicobacter, 10, 157-171.

Gong, E.J., Yun, S.C., Jung, H.Y., Lim, H., Choi, K.S., Ahn, J.Y., Lee, J.H., Kim, D.H., Choi, K.D., Song, H.J., Lee, G.H. \& Kim, J.H. (2014) Meta-analysis of first-line triple therapy for helicobacter pylori eradication in Korea: is it time to change? J. Korean Med. Sci., 29, 704-713.

Graham, D.Y. \& Fischbach, L. (2010) Helicobacter pylori treatment in the era of increasing antibiotic resistance. Gut, 59, 1143-1153.

Graham, D.Y., Hammoud, F., El-Zimaity, H.M., Kim, J.G., Osato, M.S. \& El-Serag, H.B. (2003) Meta-analysis: proton pump inhibitor or H2-receptor antagonist for Helicobacter pylori eradication. Aliment. Pharmacol. Ther, 17, 1229-1236.

Hooi, J.K.Y., Lai, W.Y., Ng, W.K., Suen, M.M.Y., Underwood, F.E., Tanyingoh, D., Malfertheiner, P., Graham, D.Y., Wong, V.W.S., Wu, J.C.Y., Chan, F.K.L., Sung, J.J.Y., Kaplan, G.G. \& Ng, S.C. (2017) Global prevalence of Helicobacter pylori infection: systematic review and meta-analysis. Gastroenterology, 153, 420-429.

Ierardi, E., Losurdo, G., Fortezza, R.F., Principi, M., Barone, M. \& Leo, A.D. (2019) Optimizing proton pump inhibitors in Helicobacter pylori treatment: old and new tricks to improve effectiveness. World J. Gastroenterol., 25, 5097-5104.

Janssen, M.J., Laheij, R.J., de Boer, W.A. \& Jansen, J.B. (2005) Meta-analysis: the influence of pre-treatment with a proton pump inhibitor on Helicobacter pylori eradication. Aliment. Pharmacol. Ther, 21, 341-345.

Jenks, P.J. (2002) Causes of failure of eradication of Helicobacter pylori. $B M J$, 325, 3-4.

Jonaitis, L., Pellicano, R. \& Kupcinskas, L. (2018) Helicobacter pylori and nonmalignant upper gastrointestinal diseases. Helicobacter, 23 Suppl 1, e12522. 
Jung, Y.S., Park, C.H., Park, J.H., Nam, E. \& Lee, H.L. (2017) Efficacy of Helicobacter pylori eradication therapies in Korea: a systematic review and network meta-analysis. Helicobacter, 22, e12389.

Kantor, E.D., O'Connell, K., Du, M., Mendelsohn, R.B., Liang, P.S. \& Braunstein, L.Z. (2021) Ranitidine use and cancer risk: results from UK Biobank. Gastroenterology, 160, 1856-1859 e1855.

Kim, S.E., Park, M.I., Park, S.J., Moon, W., Choi, Y.J., Cheon, J.H., Kwon, H.J., Ku, K.H., Yoo, C.H., Kim, J.H., Lee, G.W. \& Song, S.E. (2015) Trends in Helicobacter pylori eradication rates by first-line triple therapy and related factors in eradication therapy. Korean J. Intern. Med., 30, 801-807.

Kim, S.G., Jung, H.K., Lee, H.L., Jang, J.Y., Lee, H., Kim, C.G., Shin, W.G., Shin, E.S. \& Lee, Y.C.; Korean College of Helicobacter and Upper Gastrointestinal Research (2014) Guidelines for the diagnosis and treatment of Helicobacter pylori infection in Korea, 2013 revised edition. J. Gastroenterol. Hepatol., 29, 1371-1386.

Ko, S.W., Kim, Y.J., Chung, W.C. \& Lee, S.J. (2019) Bismuth supplements as the first-line regimen for Helicobacter pylori eradication therapy: systemic review and meta-analysis. Helicobacter, 24, e12565.

Kotilea, K., Mekhael, J., Salame, A., Mahler, T., Miendje-Deyi, V.Y., Cadranel, S. \& Bontems, P. (2017) Eradication rate of Helicobacter Pylori infection is directly influenced by adherence to therapy in children. Helicobacter, 22, e12383.

Krzyzek, P. \& Grande, R. (2020) Transformation of Helicobacter pylori into coccoid forms as a challenge for research determining activity of antimicrobial substances. Pathogens, 9, 184.

Labenz, J., Gyenes, E., Ruhl, G.H. \& Borsch, G. (1993) Omeprazole plus amoxicillin: efficacy of various treatment regimens to eradicate Helicobacter pylori. Am. J. Gastroenterol., 88, 491-495.

Labenz, J., Stolte, M., Blum, A.L., Jorias, I., Leverkus, F., Sollbohmer, M., Bertrams, J. \& Borsch, G. (1995) Intragastric acidity as a predictor of the success of Helicobacter pylori eradication: a study in peptic ulcer patients with omeprazole and amoxicillin. Gut, 37, 39-43.

Lee, J.W., Kim, N., Kim, J.M., Nam, R.H., Chang, H., Kim, J.Y., Shin, C.M., Park, Y.S., Lee, D.H. \& Jung, H.C. (2013) Prevalence of primary and secondary antimicrobial resistance of Helicobacter pylori in Korea from 2003 through 2012. Helicobacter, 18, 206-214.

Malfertheiner, P., Megraud, F., O'Morain, C.A., Gisbert, J.P., Kuipers, E.J., Axon, A.T., Bazzoli, F., Gasbarrini, A., Atherton, J., Graham, D.Y., Hunt, R., Moayyedi, P., Rokkas, T., Rugge, M., Selgrad, M., et al. (2017) Management of Helicobacter pylori infection-the Maastricht V/Florence Consensus Report. Gut, 66, 6-30.
McColm, A.A., McLaren, A., Klinkert, G., Francis, M.R., Connolly, P.C., Grinham, C.J., Campbell, C.J., Selway, S. \& Williamson, R. (1996) Ranitidine bismuth citrate: a novel anti-ulcer agent with different physico-chemical characteristics and improved biological activity to a bismuth citrateranitidine admixture. Aliment. Pharmacol. Ther, 10, 241-250.

O’Connor, A., Liou, J.M., Gisbert, J.P. \& O'Morain, C. (2019) Review: treatment of Helicobacter pylori infection 2019. Helicobacter, 24 Suppl 1, e12640.

Saniee, P., Shahreza, S. \& Siavoshi, F. (2016) Negative effect of Proton-pump Inhibitors (PPIs) on Helicobacter pylori growth, morphology, and urease test and recovery after PPI removal-an in vitro study. Helicobacter, 21, 143-152.

Savoldi, A., Carrara, E., Graham, D.Y., Conti, M. \& Tacconelli, E. (2018) Prevalence of antibiotic resistance in Helicobacter pylori: a systematic review and meta-analysis in World Health Organization regions. Gastroenterology, 155, 1372-1382 e1317.

Shin, W.G., Lee, S.W., Baik, G.H., Huh, K.C., Lee, S.I., Chung, J.W., Jung, W.T., Park, M.I., Jung, H.K., Kim, H.U., Kim, J.H., Seol, S.Y., Yoon, S.M., Jeon, S.W., Hong, S.J., et al. (2016) Eradication rates of Helicobacter pylori in Korea over the past 10 years and correlation of the amount of antibiotics use: nationwide survey. Helicobacter, 21, 266-278.

Sonnenberg, A. \& Genta, R.M. (2013) Helicobacter pylori is a risk factor for colonic neoplasms. Am. J. Gastroenterol., 108, 208-215.

Stables, R., Campbell, C.J., Clayton, N.M., Clitherow, J.W., Grinham, C.J., McColm, A.A., McLaren, A. \& Trevethick, M.A. (1993) Gastric anti-secretory, mucosal protective, antipepsin and anti-Helicobacter properties of ranitidine bismuth citrate. Aliment. Pharmacol. Ther., 7, 237-246.

Tokoro, C., Inamori, M., Koide, T., Sekino, Y., Iida, H., Sakamoto, Y., Endo, H., Hosono, K., Takahashi, H., Yoneda, M., Yasuzaki, H., Ogawa, M., Abe, Y., Kubota, K., Saito, S., et al. (2011) Influence of pretreatment with $\mathrm{H} 2$ receptor antagonists on the cure rates of Helicobacter pylori eradication. Med. Sci. Monit., 17, CR235-240.

Trikudanathan, G., Philip, A., Dasanu, C.A. \& Baker, W.L. (2011) Association between Helicobacter pylori infection and pancreatic cancer. A cumulative meta-analysis. JOP, 12, 26-31.

Yoon, H.J., Kim, J.H., Seo, G.H. \& Park, H. (2021) Risk of cancer following the Use of N-Nitrosodimethylamine (NDMA) contaminated ranitidine products: a nationwide cohort study in South Korea. J. Clin. Med., 10, 153.

Yoon, S.B., Park, J.M., Lee, J.Y., Baeg, M.K., Lim, C.H., Kim, J.S., Cho, Y.K., Lee, I.S., Kim, S.W. \& Choi, M.G. (2014) Longterm pretreatment with proton pump inhibitor and Helicobacter pylori eradication rates. World J. Gastroenterol., 20, 1061-1066. 